\title{
ON THE LEVEL CROSSING OF MULTI- DIMENSIONAL DELAYED RENEWAL PROCESSES
}

\author{
JEWGENI H. DSHALALOW \\ Department of Applied Mathematics \\ Florida Institute of Technology \\ Melbourne, FL 32901, USA \\ eugene@winnie.fit.edu
}

(Received February 1997; Revised August, 1997)

\begin{abstract}
The paper studies the behavior of an $(l+3)$ th-dimensional, delayed renewal process with dependent components, the first three (called active) of which are to cross one of their respective thresholds. More specifically, the crossing takes place when at least one of the active components reaches or exceeds its assigned level. The values of the other two active components, as well as the rest of the components (passive), are to be registered. The analysis yields the joint functional of the "crossing level" and other characteristics (some of which can be interpreted as the first passage time) in a closed form, refining earlier results of the author. A brief, informal discussion of various applications to stochastic models is presented.
\end{abstract}

Key words: Fluctuations, first excess level, first passage time, termination index, renewal process, marked point process, N-policy queues, D-policy queues, queues with vacations.

AMS subject classifications: $60 \mathrm{~K} 05,60 \mathrm{~K} 10,60 \mathrm{~K} 25,60 \mathrm{G} 25,68 \mathrm{M} 20$, 90A09, 90A60, 90B22

\section{Introduction}

The behavior of sums of independent random variables about a fixed level is a classical theme in the theory of fluctuations. In various applied probability models, the information about the level crossing and first passage time of the sum of random variables is of significant importance. In the case of nonnegative random variables, one can derive tame analytic formulas for corresponding functionals of the "first excess level" and first passage time [1].

In a basic scenario, we consider a marked delayed renewal process with dependent marking, so that the marks, as the marginal process, also form a delayed renewal process. Generally speaking, it is a two-dimensional renewal process, say $\left(\tau_{n}, M_{n}\right)$ with mutually dependent components, the first of which represents some events on the time axis. The marked process $M_{n}$ (which can be both discrete- or continuous- 
valued) evolves until it crosses a fixed, non-negative threshold upon one of the events $\tau_{n}$. Consequently, this component process is called active, while the other component $\tau_{n}$ is passive.

A common application is queueing with $\mathrm{N}$-policy and vacations (see Heyman [8], Lee et al. [9-11], Loris-Teghem [13-14], and Muh [15]). Here, when the system is exhausted, the server leaves on vacation, which includes a series of individual vacation trips or segments. The server resumes his main work when, upon completion of one of the vacation trips, the buffer accumulates to $N$ or more units. (This policy is aimed to reduce unwanted switchovers between busy and idle modes.) Another queueing application is D-policy (see Balachandran and Tijms [2], Dshalalow [7], and Li and Niu [12]). In this case, the idle or vacationing server gets back to service when the accumulated work, in terms of the cumulative service time, hits a specified level $D$. A combination of the two policies, suggested and studied in Dshalalow [5,6], yielded yet another practical application; the server would resume his primary work when the number of units in the buffer or the cumulative work hit their respective levels, whichever of the two comes first. This is associated with a three-dimensional renewal process, say $\left(\tau_{n}, Q_{n}, W_{n}\right)$, of which the first component is passive and the other two (queue length and workload) are active.

Other applications may be found in the stock market. For example, this analysis can predict the first fall of a stock from a bull spree (or visa versa), as well as its level, by introducing an auxiliary process, whose increments are zeros or ones, dependent on whether or not the stock gained or lost in successive trades.

This paper deals with a further refinement of the studies initiated by the author in $[3,4]$. It seemed reasonable to extend the number of active and passive components, and as it turns out, the outgoing functionals of three active and a finitely many passive components still yield compact formulas of a closed analytic form. The stock market can make use of the results, especially in individual, diversified packages or mutual funds dominated by two or three major stocks. Here it is worthwhile to predict the first drop of any major components of the package. Inventories and queueing may also find some practical applications of these results. For instance, we may consider (batches of) units entering a servicing system along with their weights, volumes, lengths, or energy. The service facility would stop vacationing when the queue length, workload, or cumulative length of all units in the system crosses its respective threshold, whichever comes first. This paper deals exclusively with the derivation of formulas for the functionals of the values of a general $(l+3)$ th-dimensional, delayed renewal process (with all dependent components) registered at the moment one of the three active components crosses its assigned threshold. Applications, like those mentioned above, will follow.

\section{Preliminaries}

Suppose $\Re_{R}=\Re_{m}=\left(A_{m}, B_{m}, C_{m}, \tau_{m}^{1}, \ldots, \tau_{m}^{l} ; m=0,1, \ldots\right)$ is an $(l+3)$ th-dimensional, delayed renewal process defined on the probability space $(\Omega, \mathcal{A}, P)$. In other words, the vector increments $\Re_{m}-\Re_{m-1}=\left(X_{m}, Y_{m}, Z_{m}, \Delta_{m}^{1}, \ldots, \Delta_{m}^{l}\right), m=0,1, \ldots\left(\mathscr{R}_{-1}=\right.$ 0), are independent (although their components are not), and for $m \geq 1$, identically jointly distributed.

Without loss of generality, we assume that the first three components are discrete, each valued in $\{0,1, \ldots\}$, and the other $l$ are continuous, each valued in $\mathbb{R}_{+}$. 
The joint distribution of the above increments is assumed to be given in terms of the following functionals:

$$
\gamma_{0}(u, v, w, \boldsymbol{\theta})=\mathbb{E}\left[u^{X_{0}} v^{Y}{ }_{0} w^{Z} 0_{e}-\boldsymbol{\theta \Delta}\right]
$$

where $\boldsymbol{\theta} \Delta_{0}$ is the scalar product of $\boldsymbol{\theta}=\left(\theta_{1}, \ldots, \theta_{l}\right)$ and $\Delta_{0}=\left(\Delta_{0}^{1}, \ldots, \Delta_{0}^{l}\right)^{\mathrm{T}}$ and

$$
\gamma(u, v, w, \theta)=\mathbb{E}\left[u^{X_{1}} v^{Y_{1}} w^{Z_{1}} e^{-\boldsymbol{\theta} \Delta_{1}}\right]
$$

where $\boldsymbol{\theta} \boldsymbol{\Delta}_{1}$ is the scalar product of $\boldsymbol{\theta}=\left(\theta_{1}, \ldots, \theta_{l}\right)$ and $\Delta_{1}=\left(\Delta_{1}^{1}, \ldots, \Delta_{1}^{l}\right)^{\mathrm{T}}$.

Given non-negative integers $p, q$, and $r$, define the random variable

$$
\nu=\min \left\{\nu_{1}=\inf \left\{i: A_{i}>p\right\}, \nu_{2}=\inf \left\{j: B_{j}>q\right\}, \nu_{3}=\inf \left\{k: C_{k}>r\right\}\right\}
$$

and call it the termination index. The process $\mathscr{B}_{0}$ will evolve until one of its first three components, $A, B$, or $C$, called active components, crosses one of their respective thresholds, $p, q$, or $r$. Consequently, the remaining $l$ components of $\mathscr{B}$ are referred to as passive components. In summary, the process $\Re$ will be terminated (passing $\nu$ "phases") once one of the active components hits $p, q$, or $r$, whichever comes first. The random vector $\Re_{\nu}$ is said to be the termination level, or alternatively, the first excess level, although most likely only one of the three active components will exceed $p, q$, or $r$.

Note that if one of the entries of $\tau_{m}=\left(\tau_{m}^{1}, \ldots, \tau_{m}^{l}\right)^{\mathrm{T}}$, say $\tau_{m}^{1}$, represents time, then $\tau_{\nu}^{1}$ is referred to as the first passage time of $\mathscr{B}$.

We will be interested in a closed form for the functional

$$
\mathbb{E}\left[\xi^{\nu} u^{A} \nu_{v}^{B} \nu_{w}^{C} \nu_{e}^{-\boldsymbol{\theta} \tau} \nu\right]
$$

where $\theta \tau_{\nu}$ is the scalar product of vectors $\theta$ and $\tau_{\nu}$.

For functions $f: \mathbb{N}_{0} \rightarrow \mathbb{R}$ and $g: \mathbb{N}_{0}^{3} \rightarrow \mathbb{R}$, define the transformations

and

$$
D_{j} f(w)=(1-w) \sum_{j \geq 0} w^{j} f(j),|w|<1,
$$

$$
D_{h, i, s} g(x, y, z)=D_{h} D_{i} D_{s} g(x, y, z)
$$

The functions $f$ and $g$ can be restored through the inverse operator

$$
\Im_{x}^{k}(\cdot)=\left\{\begin{array}{cc}
\lim _{x \rightarrow 0} \frac{1}{k !} \frac{\partial^{k}}{\partial x^{k}} \frac{1}{1-x}(\cdot), & k \geq 0 \\
0, & k=0
\end{array}\right.
$$

used once:

$$
\mathfrak{T}_{x}^{k}\left(D_{s} f(x)\right)=f(k)
$$

or repeatedly, for instance:

$$
\mathfrak{D}_{x}^{p} \varpi_{y}^{q} \varpi_{z}^{r}\left(D_{h, i, s} g(x, y, z)\right)=g(p, q, r)
$$

Denote 


$$
\mathscr{F}=\mathscr{F}(\xi, u, v, w, \boldsymbol{\theta} ; x, y, z)=D_{h, i, s} \mathbb{E}\left[\xi^{\nu} u^{A} \nu_{v}^{B} \nu_{w}{ }^{C} e^{-\boldsymbol{\theta} \boldsymbol{\tau}} \boldsymbol{\nu}\right](x, y, z) .
$$

Theorem 1. The functional $\mathrm{F}$ satisfies the following expression:

$$
\mathscr{F}=\gamma_{0}(u, v, w, \boldsymbol{\theta})-\gamma_{0}(u x, v y, w z, \boldsymbol{\theta}) \frac{1-\xi \gamma(u, v, w, \boldsymbol{\theta})}{1-\xi \gamma(u x, v y, w z, \boldsymbol{\theta})}
$$

Proof. Obviously the events

$$
\begin{array}{lll}
H_{1}=\left\{\nu_{1}<\nu_{2}<\nu_{3}\right\}, & H_{2}=\left\{\nu_{1}<\nu_{3}<\nu_{2}\right\}, & H_{3}=\left\{\nu_{2}<\nu_{1}<\nu_{3}\right\}, \\
H_{4}=\left\{\nu_{2}<\nu_{3}<\nu_{1}\right\}, & H_{5}=\left\{\nu_{3}<\nu_{2}<\nu_{1}\right\}, & H_{6}=\left\{\nu_{3}<\nu_{1}<\nu_{2}\right\}, \\
H_{7}=\left\{\nu_{1}=\nu_{2}<\nu_{3}\right\}, & H_{8}=\left\{\nu_{1}=\nu_{3}<\nu_{2}\right\}, & H_{9}=\left\{\nu_{2}=\nu_{3}<\nu_{1}\right\}, \\
H_{10}=\left\{\nu_{1}<\nu_{2}=\nu_{3}\right\}, & H_{11}=\left\{\nu_{2}<\nu_{1}=\nu_{3}\right\}, & H_{12}=\left\{\nu_{3}<\nu_{1}=\nu_{2}\right\}, \\
H_{13}=\left\{\nu_{1}=\nu_{2}=\nu_{3}\right\} & &
\end{array}
$$

form a measurable partition of $\Omega$. Consequently, $\sum_{i=1}^{13} I_{H_{i}}=1$ and $\mathscr{F}=\sum_{i=1}^{13} \mathcal{F}_{i}$, where $I_{H}$ is the indicator function of set $H$ and

$$
\mathscr{F}_{i}=\mathscr{F}_{i}(\xi, u, v, w, \boldsymbol{\theta} ; x, y, z)=D_{h, i, s} \mathbb{E}\left[\xi^{\nu} u^{A} \nu_{v}{ }^{B} \nu_{w}{ }^{C} \nu_{e}-\boldsymbol{\theta} \tau_{\nu} I_{H}\right](x, y, z) .
$$

Set $A_{-1}=B_{-1}=C_{-1}=0$.

From Dshalalow [4],

$$
\begin{aligned}
& D_{h}\left(I_{\left\{\nu_{1}=j\right\}}\right)(x)=x^{A} j-1-x^{A} j, j=0,1, \ldots, \\
& D_{i}\left(I_{\left\{\nu_{2}=k\right\}}\right)(y)=y^{B} k-1-y^{B} k, k=0,1, \ldots \text {, } \\
& D_{s}\left(I_{\left\{\nu_{3}=n\right\}}\right)(z)=z^{C_{n-1}}-z^{C_{n}, n=0,1, \ldots .}
\end{aligned}
$$

By Fubini's theorem,

$$
\mathscr{F}_{1}=\sum_{j \geq 0} \sum_{k>j} \sum_{n>k} \xi^{j} \mathbb{E}\left[R_{j k n}\right]
$$

with

$$
R_{j k n}=R_{1 j} R_{2 j} R_{3 j k} R_{4 k} R_{5 k n} R_{6 n}
$$

and

$$
\begin{aligned}
& R_{1 j}=(u x)^{A} j-1(v y)^{B} j-1(w z)^{C} j-1 e^{-\theta \tau} j-1, \\
& R_{2 j}=\left[u^{X}-(u x)^{X}\right](v y)^{Y} j_{(w z)}{ }^{Z} j_{e}{ }^{-\theta \Delta} j, \\
& R_{3 j k}=y^{Y}{ }^{Y+1} z^{Z} j+1 \ldots y^{Y} k-1 z^{Z} k-1, \\
& R_{4 k}=z^{Z} k-y^{Y} k_{z}{ }^{Z} \\
& R_{5 k n}=z^{Z} k+1 \ldots z^{Z} n-1 \text {, } \\
& R_{6 n}=1-z^{Z} n
\end{aligned}
$$

being independent, we arrive at

$$
\mathscr{F}_{1}=\frac{\gamma_{3}(z)-\gamma_{23}(y, z)}{1-\gamma_{23}(y, z)}\left\{\gamma_{0}^{1}-\gamma_{0}+\gamma_{0} \xi \frac{\gamma^{1}-\gamma}{1-\xi \gamma}\right\},
$$


where we denoted

and

$$
\begin{gathered}
\gamma=\gamma(u x, v y, w z, \boldsymbol{\theta}), \gamma_{0}=\gamma_{0}(u x, v y, w z, \boldsymbol{\theta}) \\
\gamma^{1}=\gamma(u, v y, w z, \boldsymbol{\theta}), \gamma_{0}^{1}=\gamma_{0}(u, v y, w z, \boldsymbol{\theta}) \\
\gamma_{3}(z)=\gamma(1,1, z, 0), \gamma_{23}(y, z)=\gamma(1, y, z, 0),
\end{gathered}
$$

which are the corresponding functionals of the marginal processes.

Now, $\mathcal{F}_{2}$ can be obtained by interchanging the roles of $v$ with $w$ and $y$ with $z$ :

$$
\mathscr{F}_{2}=\frac{\gamma_{2}(y)-\gamma_{23}(y, z)}{1-\gamma_{23}(y, z)}\left\{\gamma_{0}^{1}-\gamma_{0}+\gamma_{0} \xi \frac{\gamma^{1}-\gamma}{1-\xi \gamma}\right\},
$$

with

$$
\gamma_{2}(y)=\gamma(1, y, 1,0) \text {. }
$$

Analogously,

with

$$
\begin{aligned}
& \mathscr{F}_{3}=\frac{\gamma_{3}(z)-\gamma_{13}(x, z)}{1-\gamma_{13}(x, z)}\left\{\gamma_{0}^{2}-\gamma_{0}+\gamma_{0} \xi \frac{\gamma^{2}-\gamma}{1-\xi \gamma}\right\}, \\
& \mathscr{F}_{4}=\frac{\gamma_{1}(x)-\gamma_{13}(x, z)}{1-\gamma_{13}(x, z)}\left\{\gamma_{0}^{2}-\gamma_{0}+\gamma_{0} \xi \frac{\gamma^{2}-\gamma}{1-\xi \gamma}\right\}, \\
& \mathscr{F}_{5}=\frac{\gamma_{1}(x)-\gamma_{12}(x, y)}{1-\gamma_{12}(x, y)}\left\{\gamma_{0}^{3}-\gamma_{0}+\gamma_{0} \xi \frac{\gamma^{3}-\gamma}{1-\xi \gamma}\right\}, \\
& \mathscr{F}_{6}=\frac{\gamma_{2}(y)-\gamma_{12}(x, y)}{1-\gamma_{12}(x, y)}\left\{\gamma_{0}^{3}-\gamma_{0}+\gamma_{0} \xi \frac{\gamma^{3}-\gamma}{1-\xi \gamma}\right\},
\end{aligned}
$$

$$
\begin{aligned}
& \gamma^{2}=\gamma(u x, v, w z, \boldsymbol{\theta}), \gamma_{0}^{2}=\gamma_{0}(u x, v, w z, \boldsymbol{\theta}), \\
& \gamma^{3}=\gamma(u x, v y, w, \boldsymbol{\theta}), \gamma_{0}^{3}=\gamma_{0}(u x, v y, w, \boldsymbol{\theta}),
\end{aligned}
$$

and

$$
\gamma_{1}(x)=\gamma(x, 1,1,0), \gamma_{12}(x, y)=\gamma(x, y, 1,0), \gamma_{13}(x, z)=\gamma(x, 1, z, 0) .
$$

The next three $\mathcal{F}_{i}$ 's are as follows:

$$
\mathscr{F}_{7}=\sum_{k \geq 0} \sum_{n>k} D_{h, i, s} \mathbb{E}\left[\xi^{k} u^{A} k_{v}^{B} k_{w}{ }^{C} k_{e}-\boldsymbol{\theta} \tau_{k} I_{\left\{\nu_{1}=k\right\}} I_{\left\{\nu_{2}=k\right\}} I_{\left\{\nu_{3}=n\right\}}\right](x, y, z),
$$

that yields

$$
\mathscr{F}_{7}=\gamma_{0}+\gamma_{0}^{12}-\gamma_{0}^{1}-\gamma_{0}^{2}+\left(\gamma+\gamma^{12}-\gamma^{1}-\gamma^{2}\right) \gamma_{0} \xi \frac{1}{1-\xi \gamma}
$$

where

$$
\gamma^{12}=\gamma(u, v, w z, \boldsymbol{\theta}), \gamma_{0}^{12}=\gamma_{0}(u, v, w z, \boldsymbol{\theta})
$$

Similarly,

$$
\mathcal{F}_{8}=\gamma_{0}+\gamma_{0}^{13}-\gamma_{0}^{1}-\gamma_{0}^{3}+\left(\gamma+\gamma^{13}-\gamma^{1}-\gamma^{3}\right) \gamma_{0} \xi \frac{1}{1-\xi \gamma}
$$

where

and

$$
\gamma^{13}=\gamma(u, v y, w, \boldsymbol{\theta}), \gamma_{0}^{13}=\gamma_{0}(u, v y, w, \boldsymbol{\theta})
$$

$$
\mathcal{F}_{9}=\gamma_{0}+\gamma_{0}^{23}-\gamma_{0}^{2}-\gamma_{0}^{3}+\left(\gamma+\gamma^{23}-\gamma^{2}-\gamma^{3}\right) \gamma_{0} \xi \frac{1}{1-\xi \gamma}
$$

where 


$$
\gamma^{23}=\gamma(u, v y, w, \boldsymbol{\theta}), \gamma_{0}^{23}=\gamma_{0}(u, v y, w, \boldsymbol{\theta}) .
$$

The rest of $\mathscr{F}_{i}$ are subject to quite analogous calculations:

$$
\begin{gathered}
\mathscr{F}_{10}=\frac{1-\gamma_{2}(y)-\gamma_{3}(z)+\gamma_{23}(y, z)}{1-\gamma_{23}(y, z)}\left\{\gamma_{0}^{1}-\gamma_{0}+\gamma_{0} \xi \frac{\gamma^{1}-\gamma}{1-\xi \gamma}\right\}, \\
\mathscr{F}_{11}=\frac{1-\gamma_{1}(x)-\gamma_{3}(z)+\gamma_{13}(x, z)}{1-\gamma_{13}(x, z)}\left\{\gamma_{0}^{2}-\gamma_{0}+\gamma_{0} \xi \frac{\gamma^{2}-\gamma}{1-\xi \gamma}\right\}, \\
\mathscr{F}_{12}=\frac{1-\gamma_{1}(x)-\gamma_{2}(z)+\gamma_{12}(x, z)}{1-\gamma_{12}(x, z)}\left\{\gamma_{0}^{3}-\gamma_{0}+\gamma_{0} \xi \frac{\gamma^{3}-\gamma}{1-\xi \gamma}\right\}, \\
\mathscr{F}_{13}=\gamma_{0}^{123}-\gamma_{0}^{13}-\gamma_{0}-\gamma_{0}^{12}-\gamma_{0}^{23}+\gamma_{0}^{1}+\gamma_{0}^{2}+\gamma_{0}^{3} \\
+\left(\gamma^{123}+\gamma^{1}+\gamma^{2}+\gamma^{3}-\gamma^{12}-\gamma^{13}-\gamma^{23}-\gamma\right) \gamma_{0} \xi \frac{1}{1-\xi \gamma},
\end{gathered}
$$

where

$$
\gamma^{123}=\gamma(u, v, w, \theta) \text { and } \gamma_{0}^{123}=\gamma_{0}(u, v, w, \theta) .
$$

The summation of $\mathcal{F}_{1}$ through $\mathscr{F}_{13}$ yields formula (11).

Now, the functional $\mathbb{E}\left[\xi^{\nu} u^{A} \nu_{v}{ }^{B} \nu_{w}{ }^{C} e^{-\boldsymbol{\theta} \boldsymbol{\tau}}\right]$ can be restored through

$$
\begin{aligned}
& \mathbb{E}\left[\xi^{\nu} u^{A} \nu_{v}^{B} \nu_{w}^{C} \nu_{e}^{\left.-\boldsymbol{\theta} \tau_{\nu}\right]}=\mathscr{D}_{x}^{p} \mathscr{D}_{y}^{q} \mathscr{D}_{z}^{r} \varsubsetneqq\right. \\
& =\gamma_{0}(u, v, w, \boldsymbol{\theta})-[1-\xi \gamma(u, v, w, \boldsymbol{\theta})] \Phi_{x}^{p} \Phi_{y}^{q} \Phi_{z}^{r}\left\{\frac{\gamma_{0}(u x, v y, w z, \boldsymbol{\theta})}{1-\xi \gamma(u x, v y, w z, \boldsymbol{\theta})}\right\} .
\end{aligned}
$$

Analogously, we can also arrive at cases where all three active components of $\Re_{0}$ are continuous or mixed. In turn, all or some of the passive components of $\mathscr{B}$ can be discrete. For instance, let the component $C$ be continuous. Then substitute $e^{-\vartheta}$ for $w$ in $\mathbb{E}\left[\xi^{\nu} u^{A} \nu_{v}^{B} \nu_{w}{ }^{C} e^{-\boldsymbol{\theta} \tau} \nu\right], \gamma$ and $\gamma_{0}$ and use the transformation

$$
D_{\eta}^{c} f(s)=s \int_{\eta=0}^{\infty} e^{-s \eta} f(\eta) d \eta
$$

in place of $D_{h}$. The function $f$ can be restored by using the inverse Laplace-like operator $\mathcal{L}^{r}(\cdot)=\operatorname{Lapl}^{-1}\left(\frac{1}{s}(\cdot)\right)$, where $\mathrm{Lapl}^{-1}$ stands for the inverse of the Laplace transform.

Given the third active component is continuous, we have the following result.

Theorem 2. The functional

$$
\boldsymbol{F}=\boldsymbol{F}\left(\xi, u, v, e^{-\vartheta}, \boldsymbol{\theta} ; x, y, s\right)=D_{h, i} D_{\eta}^{c} \mathbb{E}\left[\xi^{\nu} u^{A} \nu_{v}^{B} \nu_{e}-\vartheta C_{\nu} e^{-\boldsymbol{\theta} \boldsymbol{\tau}} \nu\right](x, y, s)
$$

satisfies the following expression:

$$
\mathscr{F}=\gamma_{0}(u, v, \vartheta, \boldsymbol{\theta})-\gamma_{0}(u x, v y, \vartheta+s, \boldsymbol{\theta}) \frac{1-\xi \gamma(u, v, \vartheta, \boldsymbol{\theta})}{1-\xi \gamma(u x, v y, \vartheta+s, \boldsymbol{\theta})} .
$$

The proof of this theorem is almost identical to that of Theorem 1. The formula restoring the functional $\mathbb{E}\left[\xi^{\nu} u^{A} \nu_{v}{ }^{B} \nu_{w}{ }^{C} \nu_{e}-\boldsymbol{\theta} \boldsymbol{\tau}_{\nu}\right]$ is also very similar to (48): 


$$
\begin{gathered}
\mathbb{E}\left[\xi^{\nu} u^{A} \nu_{v}^{B} \nu_{e}-\vartheta C_{\nu}-\boldsymbol{\theta} \tau_{\nu}\right]=\mathscr{D}_{x}^{p} \mathscr{D}_{y}^{q} \mathscr{L}^{r} \mathscr{F}\left(\xi, u, v, e^{-\vartheta}, \boldsymbol{\theta} ; x, y, s\right) \\
=\gamma_{0}(u, v, \vartheta, \boldsymbol{\theta})-[1-\xi \gamma(u, v, \vartheta, \theta)] \mathscr{D}_{x}^{p} \varpi_{y}^{q} \mathcal{L}^{r}\left\{\frac{\gamma_{0}(u x, v y, \vartheta+s, \boldsymbol{\theta})}{1-\xi \gamma(u x, v y, \vartheta+s, \boldsymbol{\theta})}\right\} .
\end{gathered}
$$

\section{References}

[1] Abolnikov, L. and Dshalalow, J.H., A first passage problem and its applications to the analysis of a class of stochastic models, J. Appl. Math. Stoch. Analysis, 5:1 (1992), 83-98.

[2] Balachandran, K.R. and Tijms, H., On the D-policy for the $M / G / 1$ queue, Manag. Sci., 21:9 (1975), 1073-1076.

[3] Dshalalow, J.H., On termination time processes, in Studies in Applied Probability (ed. by J. Galambos and J. Gani, Essays in honor of Lajos Takács), J. Appl. Prob., Special Volume 31A (1994), 325-336.

[4] Dshalalow, J.H., Excess level processes in queueing, in: Advances in Queueing (ed. by J.H. Dshalalow), 243-262, CRC Press, Boca Raton, FL 1995.

[5] Dshalalow, J.H., On applications of excess level processes to (N,D)-policy bulk queueing systems, in Special Issue of Journ. Appl. Math. Stoch. Anal. Dedicated to the Memory of Roland L. Dobrushin (ed. by J.H. Dshalalow), 9:4 (1996), 551-562.

[6] Dshalalow, J.H., Corrections: On applications of excess level processes to (N,D)-policy bulk queueing systems, Journ. Appl. Math. Stoch. Anal., 10:2 (1997),

[7] Dshalalow, J.H., Queueing processes in bulk systems under D-policy, J. Appl. Prob., (1998, to appear).

[8] Heyman, D.P., Optimal operating policies for $M / G / 1$ queueing systems, Oper. Res. 16:2 (1968), 362-382.

[9] Lee, H.W., Lee, S.S., and Chae, K.C., Operating characteristics of $M^{X} / G / 1$ queue with N-policy, Queueing Sys., 15 (1994), 387-399.

[10] Lee, H.W., Lee, S.S., Park, J.O., and Chae, K.C., Analysis of $M^{X} / G / 1$ queue with N-policy and multiple vacations, J. Appl. Prob., 31 (1994), 467-496.

[11] Lee, H.W., Lee, S.S., and Chae, K.C., A fixed-size batch service queue with vacations, J. Appl. Math. Stoch. Anal., 9:2 (1996), 205-219.

[12] Li, J. and Niu, S-C., The waiting time distribution for the $G I / G / 1$ queue under D-policy, Prob. Engineer. Inform. Sci., 6 (1992), 287-308.

[13] Loris-Teghem, J., Hysteretic control of an $M / G / 1$ queueing system with two service time distributions and removable server, in: Point Processes and Queueing Problems, Colloquia Mathematica Societatis János Bolyai, Hungary, 24 (1978), 291-305.

[14] Loris-Teghem, J., Imbedded and non-imbedded stationary distributions in a finite capacity queueing system with removable server, Cah. Centr. d'Etud. Rech. Opér., 26:1-2 (1984), 87-94.

[15] Muh, D.C.-R., A bulk queueing system under N-policy with bilevel service delay discipline and start-up time, J. Appl. Math. Stoch. Anal., 6:4 (1993), 359-384. 


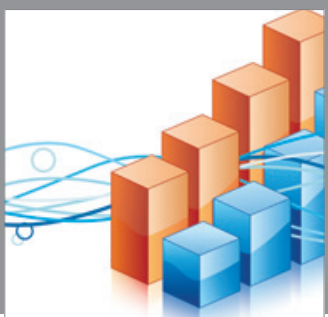

Advances in

Operations Research

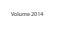

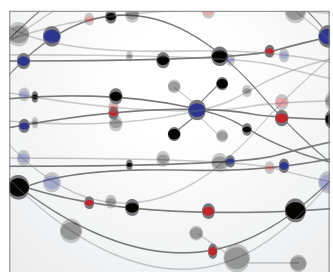

\section{The Scientific} World Journal
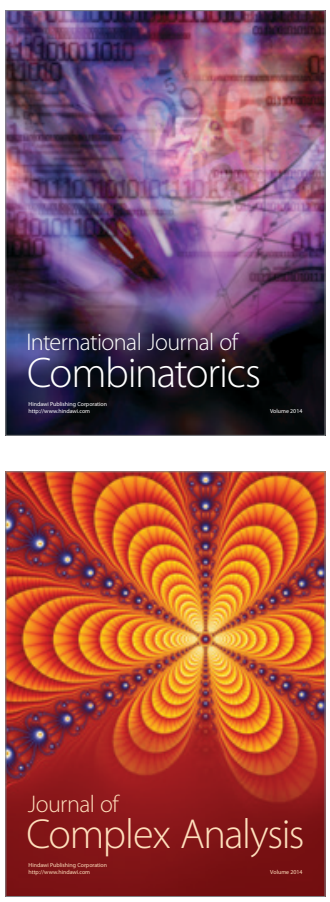

International Journal of

Mathematics and

Mathematical

Sciences
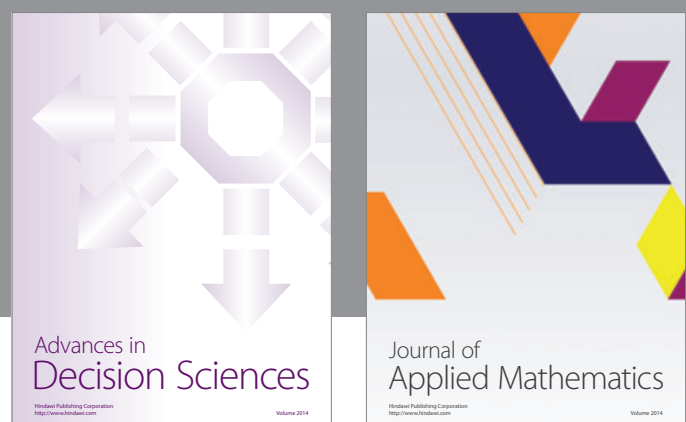

Journal of

Applied Mathematics
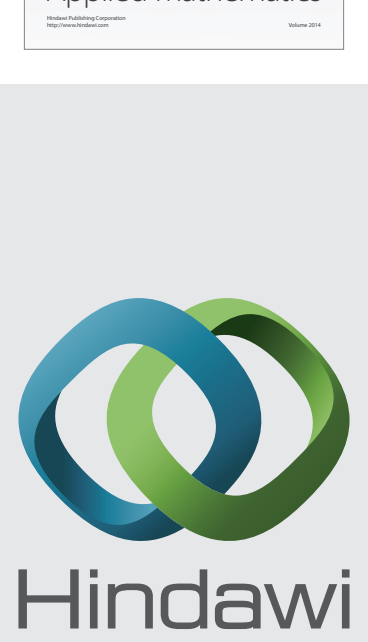

Submit your manuscripts at http://www.hindawi.com
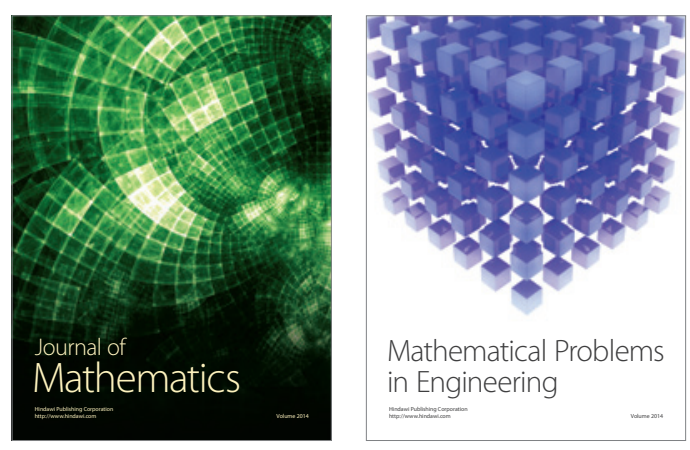

Mathematical Problems in Engineering
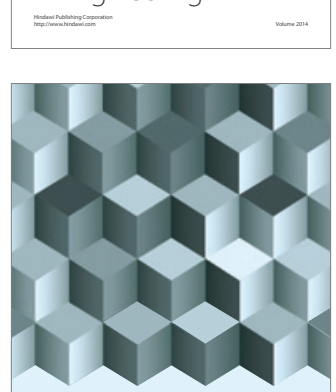

Journal of

Function Spaces
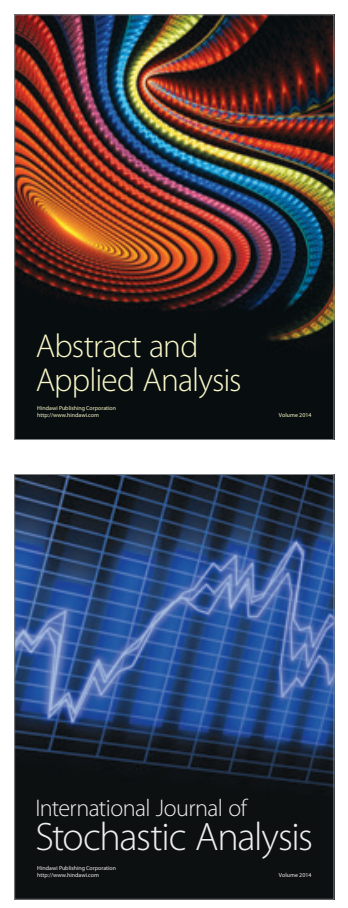

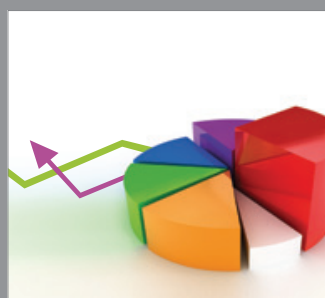

ournal of

Probability and Statistics

Promensencen
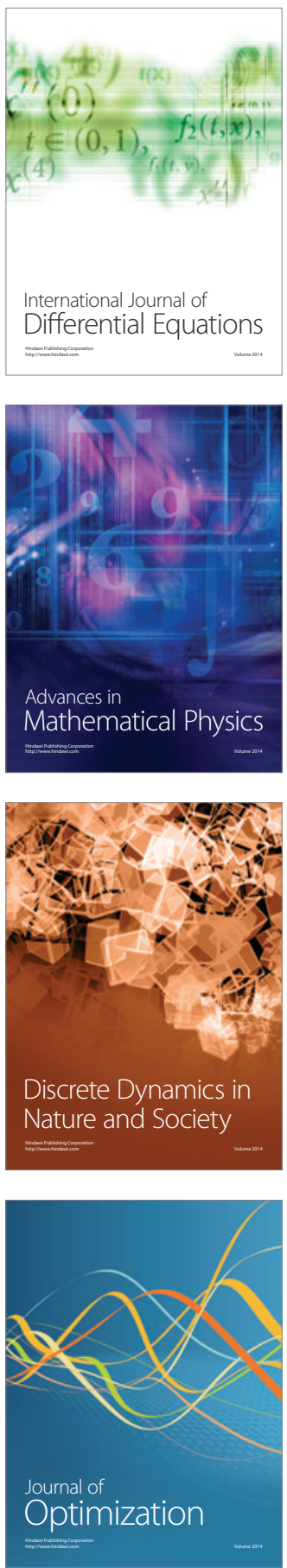